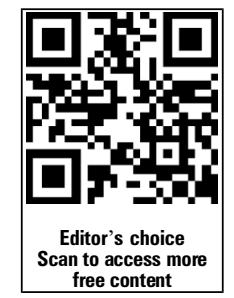

\title{
Contraception use and impact on pregnancy prevention in women participating in an HIV prevention trial in South Africa
}

\author{
Jayajothi Moodley, ${ }^{1}$ Sarita Naidoo, ${ }^{2}$ Handan Wand, ${ }^{3}$ Gita Ramjee, ${ }^{4}$ \\ and the Microbicides Development Programme team
}

For numbered affiliations see end of article.

\section{Correspondence to}

Mrs Jayajothi Moodley, HIV

Prevention Research Unit, South

African Medical Research

Council, PO Box 70380,

Overport, 4067, South Africa;

Jothi.Moodley@mrc.ac.za

Received 19 September 2014 Revised 10 June 2015

Accepted 1 July 2015

Published Online First

10 August 2015

CrossMark

To cite: Moodley J, Naidoo S, Wand $\mathrm{H}$, et al. J Fam Plann Reprod Health Care

2016;42:5-11.

\begin{abstract}
Background Unplanned pregnancy rates in South Africa are high. Effective use of contraception is therefore an essential public health intervention to prevent unplanned pregnancies. This study describes contraception use and its impact on pregnancy in women participating in HIV prevention research and its implications for public health practice.
\end{abstract}

Method A secondary analysis of sociodemographic, behavioural, contraception use, and pregnancy incidence data was conducted amongst women participating in the Microbicides Development Programme (MDP) 301 trial conducted in Durban, South Africa. Log-rank tests were carried out to compare the pregnancy incidence between women who reported use of injectable contraceptive methods compared to women using oral contraceptive pills, using condoms and other methods (intrauterine device, traditional methods and natural methods). The effect of types of contraceptives on pregnancy incidence was assessed using Cox proportional hazards regression models.

Results Of the 2018 women enrolled, injectable contraception was the most commonly used method (52\%) compared to pills, condoms for pregnancy prevention and other methods. Injectable contraception use was associated with lower crude pregnancy incidence of 4.4 per 100 woman-years [95\% confidence interval (95\% Cl 3.3-5.9)] compared to women using pills [19.3 per 100 woman-years $(95 \% \mathrm{Cl}$ 13.3-28.0)], condoms [19.7 per 100 womanyears $(95 \% \mathrm{Cl} 16.3-23.6)]$ and other methods [11.5 per 100 woman-years (95\% Cl 7.5-17.6)] This effect remained significant when adjusted for age, level of education, condom use at last sex act [hazard ratio $0.27,(95 \% \mathrm{Cl} 0.16-0.47$, $p<0.001)]$.
Key message points

- Injectable contraception use and effectiveness in pregnancy prevention remains high in South Africa.

- In high HIV endemic settings, injectable contraceptive use should be promoted together with condom use for HIV prevention.

- All family planning centres must be equipped to offer all contraceptive methods including long-acting methods such as intrauterine devices, implants and tubal ligation.

Conclusion Injectable contraception offered a high level of protection against pregnancies among women in Durban.

Trial registration number ISRCTN64716212.

\section{INTRODUCTION}

Unplanned pregnancies contribute to maternal and infant mortality and morbidity, and impact on women's social, economic and psychosocial wellbeing. ${ }^{1}$ Effective contraception coverage targeted at women with no pregnancy intention is therefore essential to improve maternal and infant health outcomes.

Pregnancy rates during typical contraceptive use represent the probability of pregnancy occurring when the contraceptive use is a mixture of perfect use and incorrect or inconsistent use. ${ }^{2}$ Known highly effective methods of contraception are injectable contraception, intrauterine contraceptive devices (IUDs), tubal ligation (TL) and implants with failure rates 
of $6.0 \%, 0.8 \%, 0.5 \%$ and $0.05 \%$, respectively, following typical use. ${ }^{3}$ The factors known to impact on contraceptive method use are socioeconomic factors, knowledge of contraception, marital status, parity, education level, partner and family expectation of fertility, knowledge about contraceptive choices, access to contraceptive services and counselling on possible side effects of contraceptive methods. ${ }^{4}$

During the period 2005-2009, the contraceptive methods available in South Africa (SA) at no cost via the South African Department of Health facilities included male and female condoms, injectable contraceptives [depot medroxyprogesterone acetate (DMPA) and norethisterone enantate (NET-EN)], combined oral contraceptive pills (COCs) $\left[\left(\right.\right.$ Triphasil $^{\circledR}$, Nordette ${ }^{\circledR}$ ) and the progesterone-only pill (POP) $\left(\right.$ Microval $\left.{ }^{\circledR}\right)$ ]. Additionally, women could access IUDs and TL at family planning centres (FPCs) offering these services. Implants were not available in SA during the period 2005-2009. The South Africa Demographic and Health Survey (SADHS) reported in 2003 that injectable contraceptives were the most commonly used method (33\%) among sexually active women. ${ }^{5}$ Reported use of other methods included COCs (12\%), male condoms as contraception (8\%), IUDs $(0.8 \%)$ and TL $(14 \%){ }^{5}$ In addition to being an established effective method, and despite the availability of other contraceptive methods, injectable contraception is the method of choice in SA. ${ }^{5}$

However, the conflicting evidence from recent analysis on the possible association of hormonal contraception (HC), in particular DMPA, with increased risk for HIV acquisition creates a public health conundrum, especially in SA, where women remain at high risk for HIV acquisition. Any decision, to either withdraw or continue DMPA use in these settings, will have to carefully consider the risk of HIV acquisition and the benefit of using these highly effective methods for pregnancy prevention. In this secondary analysis we aim to describe contraception use and its impact on pregnancy in women participating in HIV prevention research, and its implications for public health practice.

\section{METHODS}

The parent study, Microbicides Development Programme (MDP) 301 study, was an international, multicentre, randomised, double-blind, placebocontrolled trial to assess the safety and efficacy of $0.5 \%$ and $2 \%$ PRO 2000/5 gels for the prevention of vaginally acquired HIV infection. ${ }^{6}$ The study enrolled HIV-negative women from three research centres in Tanzania, Uganda and Zambia and three in SA (Durban, Mtubatuba and Johannesburg). This study, a secondary analysis, focuses on participants enrolled at three trial sites within the Durban centre of the South African Medical Research Council.

Details of the MDP 301 study methods, including the eligibility criteria and main study results, have been described elsewhere. ${ }^{7}$ Briefly, the study enrolled HIV-negative, sexually active women, aged 18 years and older (in SA), who were willing to undergo pelvic examinations, have regular urine pregnancy tests, willing to use gel regularly as instructed, willing to receive health education about condoms, and likely to have no pregnancy intention during the trial. Informed consent was obtained from all participants prior to enrolment into the study. The protocol was approved by local and international ethics and regulatory bodies in all participating countries, including the US Food and Drug Administration. For the Durban centre, ethical approval was obtained from the Biomedical Research Ethics Committee of the University of KwaZulu Natal. Approval was also obtained from the South African Medicines Control Council.

\section{Study procedures}

Study visits were scheduled every 4 weeks until study exit visit, at Week 52. At each 4-week study visit, women were provided with sufficient study gel, after a negative pregnancy test using the Quidel QuickVue ${ }^{\mathrm{TM}}$ one-step human chorionic gonadotropin urine pregnancy test kit (Quidel, San Diego, CA, USA). HIV testing with pre- and post-test HIV counselling was conducted at the screening, enrolment and at Weeks 12, 24, 40 and 52 study visits. HIV education and risk reduction counselling was provided at every study visit. Women were counselled to use condoms with every sex act and received an unrestricted supply of free condoms from the clinical research sites. At every 4-week study visit, women were asked about gel and condom use at the most recent sex act and gel applicator returns were reconciled. Women were also asked at every visit about contraception use and the method of family planning used was recorded on the case report form.

\section{Contraception counselling and provision}

Women who enrolled into the study were informed that the safety of the study gel in pregnancy was not known. Women were therefore encouraged during clinical counselling to use an effective method of contraception, although this was not mandated per protocol. The Durban trial sites provided a range of contraception options to participants, in accordance with the South African National Contraception Policy Guidelines (2001) and Essential Drug List (2003). Participants were also referred to the nearest FPCs to access contraception, if preferred. Contraception methods available at the Durban sites during the study period included injectable contraception methods (DMPA and NET-EN), COCs (Nordette, Triphasil) and the POP (Microval) for women who were breastfeeding. Trained family planning nurses and clinicians provided intensive family planning counselling to participants at each visit. Women were also referred to 
government FPCs for insertion of IUDs or TL as required. Condoms were provided to participants who chose this method for family planning with counselling to use correctly at every sex act. A contraceptive visit, either to the research site or to the government FPCs, was tracked on a contraception log. This $\log$ was a tool used by clinical staff to track contraception doses taken based on self-report and/or evidence from family planning cards, and was an alert for clinical staff to remind participants to adhere to their contraception schedule. As per protocol, selfreport of contraception use was acceptable and did not need to be verified using clinic records. Contraception doses taken were also captured on case report forms. Data for this analysis were extracted from these case report forms.

\section{Statistical analysis}

Data on types of family planning methods used were collected every 4 weeks using the sexual behaviour case report form. Participants had to indicate which of the following methods they had used for family planning: COC, diaphragm, injectable Depo-Provera ${ }^{\circledR}$, injectable Nur-Isterate ${ }^{\circledR}$, IUD, male or female condom, sterilisation, Norplant ${ }^{\circledR}$ implant, foam/jelly/ spermicide, traditional methods and natural methods. Women who were sterile or who had undergone a TL were excluded from this analysis. For the purposes of this analysis, women were categorised according to their self-reported contraceptive methods: injectable methods, COCs, condoms (male and female) and other (includes traditional methods, natural methods and IUDs). Contraceptive use in this analysis represents baseline use, as women using these methods mostly remained on these methods throughout the study period. Incident pregnancy was defined as the first positive pregnancy test after a negative pregnancy test at the previous visit. Various sociodemographic factors and behavioural characteristics were described by contraceptive groups using frequencies, percentages and Chi square $\left(\chi^{2}\right)$ tests. The log-rank test and Cox proportional hazard ratios (HRs) were used to assess the effect of contraceptive method on pregnancy rates. The proportionality of the HRs were assessed using the Stata V.12.0 ${ }^{\mathrm{TM}}$ (StataCorp LP, College Station, TX, USA) function called "estat phtest" (for individual covariates and globally).

A number of covariates such as age, level of education, number of sexual acts in the past week, and condom use at last sex act were considered in the adjusted analysis. Marital status data were not collected and included as part of this analysis. Of note was the fact that $99 \%$ of the women reported having regular sex partners.

\section{RESULTS}

A total of 2018 women enrolled at the three Durban trial sites between 2005 and 2008. Baseline characteristics of women by contraceptive groups are illustrated in Table 1. Injectable contraception was the most commonly used contraception method, with $52 \%$ using this method. Of the 1039 women using an injectable method, 816 (79\%) used DMPA and 223 (21\%) used NET-EN. Use of other contraceptive methods included condoms (31\%), COCs (7.8\%) and other methods (9.6\%). Of the women using an injectable contraception method, $48 \%$ were less than 25 years of age. Women who were unemployed and without a regular source of income were more likely to use contraceptive methods such as injectables, oral contraceptives and condoms. There was a significant relationship between level of education and type of contraception used $(p<0.01)$. Women who had three or more sex acts in the past week exhibited higher use of injectable contraception, COCs and condoms than those who had fewer sex acts. Of the women using an injectable contraception method or COCs as contraception, more than $50 \%$ also used condoms at the last sexual act.

Figure 1 illustrates condom use as a method of contraception, and use of COCs, DMPA and NET-EN at 4-weekly intervals from screening to study exit. At screening $18.4 \%$ of participants used other methods of contraception that included IUDs, traditional and natural methods. Implants and diaphragms were not available in SA during the study period. Throughout the study period the DMPA injectable method was the preferred method of contraception. Condoms as a method of contraception decreased substantially between the screening visit and 4-week assessment, and continued to gradually decrease thereafter, possibly owing to the increase in uptake of hormonal methods. Although participants switched from condoms to other more effective contraceptive methods, condom use at last sex act remained stable for HIV protection, irrespective of condom use for pregnancy prevention.

\section{Pregnancy incidence}

A total of 208 pregnancies were reported in this study. The overall pregnancy incidence was estimated as 10.8 per 100 woman-years [ $95 \%$ confidence interval (95\% CI) 9.4-12.4]. Women in this cohort had a significantly reduced risk of pregnancy incidence if they were using injectable methods [ 4.4 per 100 womanyears (95\% CI 3.3-5.9)] compared to women using COCs, condoms and other methods. Adjusting for all the significant factors listed in Table 1, the decreased risk of pregnancy associated with injectable usage remained statistically significant (Table 2).

The highest incidence of pregnancy was observed in women <25 years old (13.5 per 100 woman-years). The crude pregnancy incidence among women $>35$ years was 5.6 per 100 woman-years. High pregnancy incidence was also observed in those women with sexual debut at $<19$ years old (the crude 
Table 1 Characteristics of the women by type of contraceptive method $(n=2018)^{*}$

\begin{tabular}{|c|c|c|c|c|c|}
\hline Characteristic & $\begin{array}{l}\text { Injectables } \\
(n=1039)(51.49 \%)\end{array}$ & $\begin{array}{l}\text { Pills } \\
(n=158)(7.83 \%)\end{array}$ & $\begin{array}{l}\text { Condom } \\
(n=627)(31.07 \%)\end{array}$ & $\begin{array}{l}\text { Otherst } \\
(n=194)(9.61 \%)\end{array}$ & $p$ \\
\hline Age (years) [median (IQR)] & $25(21-32)$ & $26(23-36)$ & $26(21-36)$ & $38(23-43)$ & $<0.001 \ddagger$ \\
\hline$<25$ & $505(48.60)$ & $61(38.61)$ & $276(44.02)$ & $55(28.35)$ & \\
\hline $25-29$ & $211(20.31)$ & $36(22.78)$ & $102(16.57)$ & $15 \quad(7.73)$ & \\
\hline $30-34$ & $119(11.45)$ & $19(12.03)$ & $72(11.48)$ & $16 \quad(8.25)$ & \\
\hline $35+$ & $294(19.63)$ & $42(26.58)$ & $177(28.23)$ & $108(55.67)$ & \\
\hline Employment status & & & & & 0.050 \\
\hline Employed/regular income & $171(16.46)$ & $36(22.78)$ & $100(15.95)$ & $43(22.16)$ & \\
\hline Not employed/regular income & $868(83.54)$ & $122(77.22)$ & $527(84.05)$ & $151(77.84)$ & \\
\hline Religion & & & & & 0.100 \\
\hline Others & $653(62.85)$ & $90(56.96)$ & $358(57.10)$ & $116(59.79)$ & \\
\hline Christian & $386(37.15)$ & $68(43.04)$ & $269(42.90)$ & $78(40.21)$ & \\
\hline Level of education & & & & & 0.008 \\
\hline Secondary school or more & 337 (32.44) & $48(30.38)$ & $232(37.00)$ & $47(24.23)$ & \\
\hline Less than secondary school & $702(67.56)$ & $110(69.62)$ & $395(63.00)$ & $147(75.77)$ & \\
\hline Age at first sex (years) & & & & & 0.237 \\
\hline$<15$ & $59 \quad(5.68)$ & $9 \quad(5.70)$ & $25 \quad(3.99)$ & $15 \quad(7.73)$ & \\
\hline $15-19$ & $816(78.54)$ & $121(76.58)$ & 487 (77.67) & $140(72.16)$ & \\
\hline $20+$ & $164(15.78)$ & $28(17.72)$ & $115(18.34)$ & $39(20.10)$ & \\
\hline Number of sexual acts in past week & & & & & 0.005 \\
\hline$\leq 1$ & $279(26.85)$ & $42(26.58)$ & $191(30.46)$ & $74(38.14)$ & \\
\hline 2 & $307(29.55)$ & $40(25.32)$ & $157(25.07)$ & $58(29.90)$ & \\
\hline$\geq 3$ & $453(43.60)$ & $76(48.10)$ & $279(44.50)$ & $62(31.96)$ & \\
\hline Condom used in last sexual act & & & & & $<0.001$ \\
\hline Yes & $586(56.40)$ & $92(58.23)$ & $511(81.50)$ & $57(29.38)$ & \\
\hline No & $453(43.60)$ & $66(41.77)$ & $116(18.50)$ & $137(70.62)$ & \\
\hline Diagnosis with STIs§ & & & & & 0.934 \\
\hline Yes & $236(22.71)$ & $33(20.89)$ & $144(22.97)$ & $46(23.71)$ & \\
\hline No & $803(77.29)$ & $125(79.11)$ & $483(77.03)$ & $148(76.29)$ & \\
\hline
\end{tabular}

*Excluding those women who were sterile or who had undergone tubal ligation.

tIncludes traditional methods (oral/vaginal), intrauterine contraceptive device (only three women), etc.

$\ddagger$ Both age variables: Kruskal-Wallis non-parametric test for the median, Chi square $\left(\chi^{2}\right)$ test for age groups.

$\S C h l a m y d i a$, gonorrhoea or syphilis.

IQR, interquartile range; STI, sexually transmitted infection.

pregnancy incidence rate for those aged $<15$ years was 11.7 per 100 woman-years and for $15-$ 19 -year-olds it was 11 per 100 woman-years). The proportionality assumption was not violated in any of the models considered (global $p$ values were estimated to be 0.6936 and 0.395 for adjusted and unadjusted models, respectively).

\section{DISCUSSION}

Usage levels of injectable contraception were confirmed to be high in a clinical trial setting, with 52\% of women choosing this method compared to COCs, condoms and other methods. This finding is similar to prevalence of injectable use reported in the SADHS 2003 (2007), ${ }^{5}$ which showed that injectable contraception was the most commonly preferred contraceptive method in SA (33\%). The reported high use of injectable contraception may be attributed to the dosage form, reversibility and long dosing interval which enables covert use. ${ }^{2}$ This study also showed a high reported use of condoms (31\%) as a contraceptive method. Condom use as a contraceptive method decreased substantially between the screening visit and the 4-week assessment, and this trend was sustained over time possibly due to increased use of injectables and pills. This may be due to provision of contraception at the trial sites, intense counselling and tracking at sites, which motivated women to change and sustain use of more effective methods of contraception during the trial. Correct and consistent condom use is the only proven intervention to prevent both pregnancy and HIV acquisition. It is encouraging to note that more than $50 \%$ of women who reported to be using HC (both injectable and oral pills) also reported using condoms at the last sex act. This was most probably due to the intensive condom counselling provided to all participants as part of the HIV prevention and contraception package. Greater uptake of a more effective contraception method such as an injectable method together with condoms suggests 

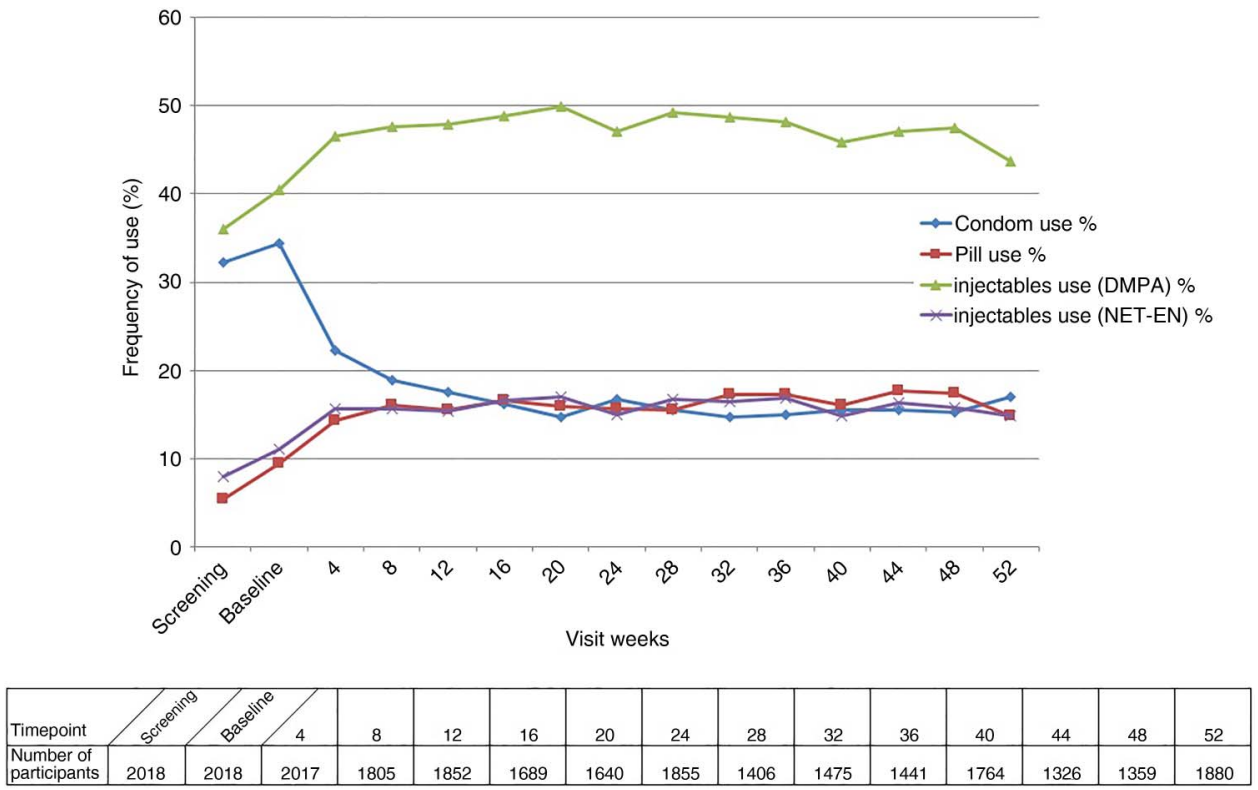

Figure 1 Contraceptive method use during the study visits. DMPA, depot medroxyprogesterone acetate; NET-EN, norethisterone acetate.

that HIV prevention was also a concern to women participating in the study. Reinforcement of dual use of condoms and an effective method of contraception in our setting therefore should be part of routine counselling provided by health care workers.

This study confirmed that $48 \%$ of the women using an injectable method were $<25$ years old. Younger women in the KwaZulu Natal region, where the Durban research centre is located, are known to be at high risk for HIV acquisition. ${ }^{8}$ A recently published paper by Wand and Ramjee found a significant association between HC use and HIV acquisition in this same cohort of MDP 301 women from the Durban sites. $^{9}$ These findings were confirmed by Crook et $a l .{ }^{10}$ who reported a modest elevated risk of HIV acquisition when analysing the MDP 301 data across all MDP research centres. Ramjee and Wand further explored the population level impact of $\mathrm{HC}$ use in this cohort. They reported that removing HC would result in $12 \%$ fewer HIV seroconversions but $72 \%$ more pregnancies. ${ }^{11}$ They concluded that the population level impact of risk of HIV infection is less than the impact of preventing unintended pregnancies and possibly newly HIV infected infants. ${ }^{11}$ These findings further underscore the need to integrate family planning and HIV prevention services to ensure women are empowered to always use dual methods for HIV and pregnancy prevention.

Our results also showed that the pregnancy incidence was higher in women with a sexual debut of $<19$ years old. This is also the same group that is at high risk for HIV acquisition due to possible high-risk behaviours. Targeting these groups at an early age, while in secondary school for contraception counselling and provision, as well as HIV risk counselling should be more consistently implemented across SA. This strategy is currently supported by the SA national contraception service delivery guidelines (2012), ${ }^{4}$ which allows the provision of contraception such as condoms, emergency contraception, oral contraceptives and injectable methods at schools, by a qualified school nurse.

As expected, we noted very low use of highly effective methods such as IUDs and male/female sterilisation. While these methods were included as part of contraception counselling, uptake of these methods by participants was low possibly because they were not provided at the clinical trial site and additionally due

Table 2 Adjusted and unadjusted hazard ratios of pregnancy

\begin{tabular}{|c|c|c|c|c|c|}
\hline $\begin{array}{l}\text { Contraceptive } \\
\text { method }\end{array}$ & $\begin{array}{l}\text { Crude pregnancy incidence } \\
(95 \% \mathrm{Cl})\end{array}$ & $\begin{array}{l}\text { Unadjusted HR } \\
(95 \% \mathrm{Cl})\end{array}$ & $p$ & $\begin{array}{l}\text { Adjusted HR } \\
(95 \% \mathrm{CI})\end{array}$ & $p$ \\
\hline Injectable & $4.4 \quad(3.3-5.9)$ & $0.38(0.23-0.64)$ & $<0.001$ & $0.27(0.16-0.47)$ & $<0.001$ \\
\hline Pills & $19.3(13.3-28.0)$ & $1.67(0.95-2.95)$ & 0.075 & $1.29(0.72-2.31)$ & 0.394 \\
\hline Condoms & $19.7(16.3-23.6)$ & $1.70(1.07-2.72)$ & 0.024 & $1.40(0.85-2.32)$ & 0.179 \\
\hline Other & $11.5(7.5-17.6)$ & 1.00 & & 1.00 & \\
\hline
\end{tabular}

The overall crude pregnancy incidence was 10.8 per 100 woman-years ( $95 \%$ Cl 9.4-12.4).

Adjusted for age, religion, number of sexual acts in the past week and condom use at last sexual act.

$95 \% \mathrm{Cl}, 95 \%$ confidence interval; HR, hazard ratio. 
to reasons of accessibility, availability and clinical staff capacity to conduct these procedures at the nearest referral facilities. Implants were not used due to their unavailability in SA. These findings highlight the urgent need to expand the contraceptive method mix in SA and to facilitate easy access to these all contraceptive options. These methods have the benefit of a long dosing interval and do not require rigorous compliance and correct use by women. ${ }^{12}$ The Department of Health in SA is currently prioritising the training of clinical staff on IUD insertion and ensuring greater availability of the IUDs at all clinical levels of care. ${ }^{12}$ The South African Medicine Control Council also recently approved the use of two implants, Jadelle ${ }^{\circledR}$ and Implanon ${ }^{\circledR}$. The Department of Health is currently facilitating training of clinical staff, and started rollout of Implanon at public sector facilities. Once these methods become more widely accessible via the Department of Health they will become part of the package of long-acting reversible contraception (LARC) available to all women. This will enable family planning providers to better expand the contraceptive method mix while ensuring that no one method dominates as a method of choice in the public sector.

While our findings confirm that use of injectable contraception remains a highly effective pregnancy prevention option, continued use of injectable contraception requires intensive counselling on dual use of condoms to protect against possible HIV acquisition. This paper adds to the current knowledge on contraceptive use in HIV prevention trials in SA. Although these results are not widely generalisable, given the high efficacy of DMPA and limited provision of other highly effective methods of contraception in this setting, these study results can be generalised to other similar low-income settings with high HIV incidence.

This study had a few limitations. Contraception use was self-reported, which is subject to recall bias. This applies to reporting the use of condoms as well. Additionally, this study was a secondary analysis of the main trial data, which was not designed to answer our research question. However, the study results do confirm that increased effort is required in this region to widen the contraceptive choices available to include LARC methods such as IUDs and implants.

\section{CONCLUSIONS}

These results confirm the high effectiveness and high usage levels of injectable contraception in this cohort of women. In light of its possible association with HIV acquisition, it is strongly recommended that women of reproductive age be provided with all available information on HC-HIV risk and a wider contraception method choice to make an informed decision regarding contraceptive use. All FPCs should be adequately equipped and have trained staff to provide access to all contraceptive methods including IUDs, referrals for TL, implants and COCs. Family planning providers must adequately counsel women and their partners that male and female condoms, if used correctly and consistently, offer protection against both HIV acquisition and pregnancy.

\section{Author affiliations}

${ }^{1}$ Senior Scientist, HIV Prevention Research Unit, South African Medical Research Council, Durban, South Africa

${ }^{2}$ Senior Scientist, HIV Prevention Research Unit, South African Medical Research Council, Durban, South Africa

${ }^{3}$ Biostatistician, The Kirby Institute, University of New South Wales, Sydney, Australia

${ }^{4}$ Chief Specialist Scientist, Director, HIV Prevention

Research Unit, South African Medical Research

Council, Durban, South Africa; and Honorary

Professor, Department of Epidemiology and Population Health, London School of Hygiene \& Tropical Medicine, London, UK

Acknowledgements The authors gratefully acknowledge the commitment of all the women who participated in the MDP301 trial and the MDP staff for their hard work and commitment. They would like to particularly thank Ramona Moodley, Brodie Daniels, Nathlee Abbai, Tarylee Reddy, Girisha Kistnasami and Fumane Molefe for their assistance in the preparation of this manuscript.

Funding The Microbicides Development Programme (MDP) is a partnership of African, UK and Spanish academic/government institutions and commercial organisations. The MDP was funded by the British Government Department for International Development (DIFD) and the UK Medical Research Council.

Competing interests None declared.

Ethics approval Biomedical Research Ethics Committee of the University of KwaZulu Natal.

Provenance and peer review Not commissioned; externally peer reviewed.

\section{REFERENCES}

1 Bafana T. Factors influencing contraceptive use and unplanned pregnancy in a South African Population. Masters dissertation, University of the Witwatersrand, Johannesburg, South Africa, 2010.

2 Hatcher RA, Trussell J, Nelson AL, et al. Contraceptive Technology (20th edn). New York, NY: Ardent Media, 2011.

3 US Department of Health and Human Services. Centers for Disease Control and Prevention. Effectiveness of Family Planning Methods. http://www.cdc.gov/reproductivehealth/ UnintendedPregnancy/PDF/Contraceptive_methods_508.pdf [accessed 1 June 2014].

4 South Africa Department of Health. National Contraception and Fertility Planning Policy and Service Delivery Guidelines. Pretoria, South Africa: Department of Health, 2012.

5 South Africa Department of Health. South Africa Demographic and Health Survey 2003. Full Report. Pretoria, South Africa: South Africa Department of Health, 2007.

6 McCormack S, Ramjee G, Kamali A, et al. PRO2000 vaginal gel for prevention of HIV-1 infection (Microbicides Development Programme 301): a phase 3, randomised, double-blind, parallel-group trial. Lancet 2010;376:1329. 
7 Nunn A, McCormack S, Crook AM, et al. Microbicides Development Programme: design of a phase III trial to measure the efficacy of the vaginal microbicide PRO 2000/5 for HIV prevention. Trials 2009;10:99.

8 South Africa Department of Health. National Antenatal Sentinel HIV and Syphilis Prevalence Survey in South Africa, 2009. Pretoria, South Africa: Department of Health, 2010.

9 Wand H, Ramjee G. The effects of injectable hormonal contraceptives on HIV seroconversion and on sexually transmitted infections. AIDS 2012;26:375-380.
10 Crook AM, Ford D, Gafos M, et al. Injectable and oral contraceptives and risk of HIV acquisition in women: an analysis of data from the MDP301 trial. Hum Reprod 2014;29:1810-1817.

11 Ramjee G, Wand H. Population-level impact of hormonal contraception on incidence of HIV infection and pregnancy in women in Durban, South Africa. Bull World Health Organ 2012;90:748-755.

12 South Africa Department of Health. National Contraception Clinical Guidelines 2012. Pretoria, South Africa: South Africa Department of Health, 2012. 